\title{
Subjective roughness and smoothness for individual judges'
}

\author{
LEROY A. STONE \\ THE UNIVERSITY OF NORTH DAKOTA
}

Ss judged a sequence of 12 emery cloths with instructions that they judge roughness and smoothness using magnitude estimation procedures. For individual Ss, power functions appropriately described the psychophysical relationships between the subjective estimations and grit numbers, however, the exponents were quite variable and were generally smaller than those reported in previous research. Reciprocality (for judged roughness-smoothness) was observed.

A half a decade ago Stevens \& Harris (1962), though not the first, conducted three experiments in which subjective roughness and smoothness were quantitatively scaled. Magnitude estimation procedures were used with 12 grits of emery cloth serving as stimuli. According to Stevens and Harris, "All three experiments gave results that were power functions of grit number with exponents in the vicinity of -1.5 for roughness and +1.5 for smoothness" (p. 494). Ekman, Hosman, \& Lindstrom (1965) used seven somewhat similar stimuli (two were identical to those used by Stevens and Harris), employed the method of ratio estimation, and utilized coefficients of friction as the physical measure. For 10 Ss they found individual exponents (the slopes of best fitting straight lines with $\log -\log$ coordinates) for roughness ranging from 0.8 to 3.5 (median $=1.80$ ) and for smoothness ranging from -0.8 to -3.0 (median $=-1.85)$. Ekman et al $(1965$, p. 24) suggested that for subjective roughness and smoothness the power function is verified for individuals but conclude that such a function "is a variable over individuals."

The purpose of the present investigation was to examine individual Ss' power function exponents for subjective roughness and smoothness based on the same stimuli and the same psychophysical method, magnitude estimation (no assigned modulus), as employed in Stevens \& Harris' (1962) Experiment 2. Method

The stimuli were new "Tri-M-ite" brand emery cloths (Grits 320, 240, 220, 180, 120, 100, 80, 60, 50, 40,30 , and 24). The same instructions and methodology as employed by Stevens and Harris in their Experiment 2 were used. One exception was that different Ss $(N=10)$ were used to estimate smoothness than those $(N=10)$ who estimated roughness. All Ss were graduate students in psychology and were quite familiar with psychophysics and psychological scaling. With the exception of one $S$, all were males.

Results

The 20 subjective scales (for individual Ss) of roughness and smoothness were plotted against the stimulus variable, grit numbers, in $\log -\log$ coordinates. Straight lines were found to offer reasonably good approximations to the data trends; i.e., power functions appropriately describe the psychophysical relationship for all Ss. For roughness, the slopes (power function exponents) were, in order: $-0.90,-0.90,-0.94,-0.95$, $-0.99,-1.24,-1.38,-1.42,-1.59$, and -1.66 (median $=-1.12)$. For smoothness, the slopes were: $0.75,0.77$, $0.79,0.81,0.90,0.91,0.97,1.11,1.26$, and 1.37 (median $=0.905$ ).

Group scales were easily obtained by computing geometric means of the individual scale values. With the group scales the slope for subjective roughness was -1.18 and for subjective smoothness, 0.95. The reciprocalities of smoothness-roughness and roughness-smoothness had slopes of -0.73 and -1.15 , respectively. The geometric mean of these two slopes is -0.91 . This averaged slope is quite near unity (reciprocality). It should be remembered that the subjective roughness and smoothness scales were based on independent Ns. The regression of the roughness scale values on the smoothness scale values may also be expressed as a correlation of -.962 ( $\mathrm{df}=10$, $\mathrm{p}<.001)$.

\section{Discussion}

The results conflict with the idea put forth by Pradhan \& Hoffman (1963), based on weight lifting experiments, that the power function does not describe data for individuals although it does reasonably represent group data. With roughness-smoothness judgments the power function does appear to apply for individuals as well as for scales based on collective judgments.

Most of the individual slopes and both group slopes were less steep than those group slopes reported by Stevens \& Harris (1962). Stevens ${ }^{2}$ has commented that this phenomenon (less steep slopes) occurs when more "noise" is present in the measurement system. No specific explanation is offered by the $E$ other than a possible casual reference to individual differences. Perhaps the Ss' familiarity with magnitude estimation methodology and the psychophysical power law influenced their magnitude estimations, although there is no research support for this view.

The variation between individual Ss' slopes appears to be substantial although not as great as reported by Ekman et al (1965). The median slopes seen in their investigation are more steep than the generalized value given by Stevens and Harris. However, because of differences with respect to stimuli and physical scale measurement, the results of these two investigations 
are not directly comparable. It is reported by Stevens and Harris that, in preliminary experiments, they found a steeper slope for roughness $(-1.5)$ than for smoothness (1.2). This same directional discrepancy also occurred with the present results. The group slopes appeared to be (although imperfectly) the reciprocal of each other (slope $=-0.91$ ).

The stimuli appeared to differ in ways other than grit size. Stevens and Harris noticed a particular nonuniformity in the manufacturing process, namely, the depth that the abrasive particles are embedded in the adhesive. Grit 240, on the group based subjective roughness scale, was judged to be more rough than Grit 220 (which had larger abrasive particles). Grit 240 , on the group subjective smoothness scale, was judged to be less smooth than Grit 220. Visual inspection (magnifying glass) of this particular stimulus (Grit 240) revealed that its grains sat higher in the adhesive than did the grains on adjacent stimuli. As a result, even though Grit 240 had smaller abrasive grains than did Grit 220 , it was judged to be rougher or less smooth. Grit 240 , based on the described sen- sory data, seemed to be structurally "defective." It may be that the defective nature of this one stimulus contributed to the lower than expected (based on previous research) power function exponents seen. Perhaps the stimuli, although they were the same grit numbers as those used by Stevens and Harris, did differ from the previously employed stimuli with respect to depth of the embedded abrasive particles.

\section{References}

EKMAN, E., HOSMAN, J., \& LINDSTROM, B. Roughness, smoothness, and preference: A study of quantitative relations in individual subjects. J. exp. Psychol, 1965, 70, 18-26.

PRADHAN, P. L., \& HOFFMAN, P. J. Effect of spacing and range of stimuli on magnitude estimation judgments. J. exp. Psychol, 1963, 66, 633-641.

STEVENS, S. S., \& HARRIS, J. R. The scaling of subjective roughness and smoothness. J. exp. Psychol, 1962, 64, 489-494.

\section{Notes}

1. This research was supported by a Public Health Service Fellowship (1-F3-MH-12,312-01) from the National Institute of Mental Health and by a grant from the University Committee on Research, The University of North Dakota.

2. S. S. Stevens, personal communication, February, 1967. 